\title{
miR-497 induces apoptosis of breast cancer cells by targeting Bcl-w
}

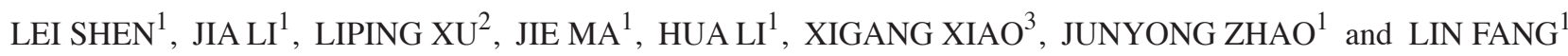 \\ ${ }^{1}$ Shanghai Tenth People's Hospital; ${ }^{2}$ Department of General Surgery, Ningbo First Hospital; \\ ${ }^{3}$ Department of General Surgery, Ningbo Second Hospital, College of Medicine, Tongji University, Shanghai, P.R. China
}

Received September 7, 2011; Accepted November 2, 2011

DOI: $10.3892 /$ etm.2011.428

\begin{abstract}
RNAs are a small class of non-coding RNAs with a critical role in the tumorigenesis and maintenance of breast cancer through binding to the 3'-untranslated regions of target mRNAs, which causes a block of translation and/or mRNA degradation. The purpose of this study was to investigate the expression of microRNA-497 (miR-497) as well as its potential role in human breast cancer. Reverse transcriptionpolymerase chain reaction (RT-PCR) was performed to determine the expression pattern of miR-497 in breast cancer and normal breast tissues. Correlation analysis was conducted to characterize the association of miR-497 expression abnormality with pathological factors. Proliferation, cell cycle and apoptosis assays were conducted to explore the potential function of miR-497 in human MCF-7 breast cancer cells. RT-PCR and Western blot analysis were employed to validate the downstream targets of miR-497. miR-497 expression was relatively decreased in breast cancer specimens and negatively correlated with TNM stage, lymphatic metastasis, tumor size and human epidermal growth factor receptor-2 $(\mathrm{P}<0.01)$. On the contrary, no correlation was found with estrogen receptor, progesterone receptor and p53 status. Functional assays revealed that miR-497 suppressed cellular growth, increased the percentage of early apoptotic cells and initiated G0/G1 cell phase arrest of MCF-7 cancer cells. RT-PCR and Western blot analysis data indicated that the overexpression of miR-497 resulted in the down-regulation of Bcl-w at the mRNA and protein levels. miR-497 may serve as a tumor suppressor gene in breast cancer. The Up-regulation of miR-497 expression causes cellular growth inhibition and apoptotic enhancement, as well as G0/ G1 phase arrest, suggesting its use as a potential therapeutic target for the treatment of breast cancer in the future.
\end{abstract}

Correspondence to: Dr Lin Fang, Department of Breast and Thyroid Surgery, Shanghai Tenth People's Hospital, Tongji University, 301 Yan Chang Road, Zhabei, Shanghai 200072, P.R. China

E-mail: fendou000@foxmail.com

Key words: breast cancer, microRNA-497, Bcl-w, cell proliferation, cell apoptosis

\section{Introduction}

Breast cancer, one of the most common female malignant tumors, is a leading cause of cancer mortality worldwide (1). Genetic mutations have been demonstrated to be causative of the tumorigenesis and maintenance of breast cancer. Over the past decades, an increasing amount of evidence has shown that microRNAs (miRNAs), a small class of non-coding RNAs, play a pivotal role in breast cancer, in which they may function as oncogenes or tumor suppressor genes (2-4).

miRNAs are derived from exons of protein-coding and non-coding genes $(5,6)$, and are then transcribed by polymerase II as a long primary transcript (primiR). Pri-miRNAs are further processed by Drosha, leading to the excision and release of approximately 70 nucleotide hairpin precursors termed pre-miRNAs (7). After being exported from the nucleus by exportin-5, the pre-miRNAs are subsequently cleaved by Dicer, releasing the $22 \mathrm{nt}$ miRNA-miRNA duplex, one strand of which in turn is incorporated into the RNA-induced silencing complex (miRISC), and eventually functions as a mature miRNA. The 'seed' region of the mature miRNA (nucleotides 2-8 at the 5' end of a miRNA) binds partially or completely to mRNA 3'-untranslated regions (3'-UTRs) of specific protein-coding genes (8). miRNAs regulate their targets by directly cleaving mRNAs or inhibiting protein synthesis, which depends on the degree of complementarity with the 3'-UTR of their targets (6). Complete complementarity between miRNAs and the 3'-UTR of their targets leads to mRNA degradation, while partial complementarity results in translation inhibition of the target protein; the latter commonly occurs in human cells (9).

There has been a large body of evidence showing the significant difference in expression of miRNAs between breast cancer and normal breast tissues $(10,11)$. These differentially expressed miRNAs are critical to the proliferation $(12,13)$, apoptosis $(14,15)$, invasion (16-19) and therapy resistance $(20,21)$ of breast cancer. In this study, miRNAs (miRs)-497, -198, -373 and -1289 were selected for expression validation in breast cancer samples. Correlation analysis was conducted to characterize the association of miR-497 expression abnormality with pathological characteristics. miR-497 mimics were used to determine the impact of miR-497 on the proliferation, apoptosis and cell cycle of breast cancer cells, as well as to determine its downstream targets. 


\section{Materials and methods}

Specimens. In this study, 48 pairs of breast cancer and normal specimens were collected from the Department of Breast and Thyroid Surgery of Shanghai Tenth People's Hospital, Shanghai, China. All the samples were confirmed as invasive ductal breast cancer, and no patients had received any chemotherapy or radiotherapy prior to surgery.

Cell culture. The MCF-7 breast cancer cell line used in this study was obtained from the Chinese Science Institute and grown in DMEM (Gibco, New York, NY, USA) supplemented with $10 \%$ fetal bovine serum (FBS) (Gibco), as well as 100 units of penicillin/ml and $100 \mu \mathrm{g}$ of streptomycin/ml (Enpromise, Hangzhou, China). Cells were incubated at $37^{\circ} \mathrm{C}$ in a humidified chamber supplemented with $5 \% \mathrm{CO}_{2}$.

Real-time polymerase chain reaction (PCR) analyses. We followed the protocol of Chen et al for primer design and real-time reverse transcription (RT)-PCR (22). The analyzed miRNAs included miR-497, miR-198, miR-373 and miR-1289. miRNAs were harvested according to the instructions of the miRcute miRNA isolation kit (Tiangen, Beijing, China). The following primers were used for the U6 small nuclear RNA, which was used as an internal control: 5'-GTCCTATCCAGT GCAGGGTCCGAGGTGCACTGGATACGACAAAATATGG AAC-3', 5'-TGCGGGTGCTCGCTTCGCAGC-3' and 5'-CCA GTGCAGGGTCCGAGGT-3'. cDNA was generated by reverse transcription according to the instructions of the PrimeScript ${ }^{\mathrm{TM}}$ RT-PCR kit (Takara, Shiga, Japan). PCR parameters for miRNA quantification were as follows: $2 \mathrm{~min}$ at $95^{\circ} \mathrm{C}$, and then 40 cycles of $30 \mathrm{sec}$ at $95^{\circ} \mathrm{C}$, and $45 \mathrm{sec}$ at $60^{\circ} \mathrm{C}$.

Total RNA was isolated using TRIzol (Invitrogen, Carlsbad, CA, USA), and cDNA was generated by reverse transcription following the protocol of the PrimeScript RT-PCR kit (Takara). Quantitative real-time PCR was performed on a 7900HT fast RT-PCR instrument (Applied Biosystems, Singapore) using SYBR-Green as the detection fluorophore. All the primers used were as follows: Bcl-w sense, CACCCAGGT CTCCGATGAAC and antisense, TTGTTGACACTCTCA GCACAC; p65 sense, GGGAAGGAACGCTGTCAGAG and antisense, TAGCCTCAGGGTACTCCATCA; Bcl-xL sense, GGTCGCATTGTGGCCTTTTTC and antisense, TGCTGCATTGTTCCCATAGAG; Bcl-2 sense, GAACTG GGGGAGGATTGTGG and antisense, CCGGTTCAGGTA CTCAGTCA; caspase-3 sense, ATGGAAGCGAATCAATGG ACTC and antisense, CTGTACCAGACCGAGATGTCA; caspase- 8 sense, CCTGTCACTGTCTTGTACCCT and antisense, CCCGCAGTATCTTGCCTCC; $\beta$-actin sense, AGC GAGCATCCCCCAAAGTT and antisense, GGGCACGAA GGCTCATCATT. The PCR parameters for relative quantification were as follows: $2 \mathrm{~min}$ at $95^{\circ} \mathrm{C}$, followed by 40 cycles of $15 \mathrm{sec}$ at $95^{\circ} \mathrm{C}$ and $30 \mathrm{sec}$ at $60^{\circ} \mathrm{C}$. Each sample was tested in triplicate. The mRNA level of $\beta$-actin was used as the internal control, and gene-specific mRNA expression was normalized against $\beta$-actin expression.

Transfection assay. Cells $\left(1 \times 10^{6}\right)$ were added into each well of a 6-well plate and cultured with DMEM medium without serum and antibiotics. As the confluency of MCF-7 breast cancer cells reached 80-90\%, miR-497 mimics and lipofect at the ratio of $1 \mu \mathrm{g}: 3 \mu \mathrm{l}$ were diluted to $250 \mu \mathrm{l}$ by DMEM medium, respectively, and incubated for $5 \mathrm{~min}$ at room temperature. miR-497 mimics and the lipofect dilution were gently combined and incubated for $20 \mathrm{~min}$. Subsequently, $500 \mu \mathrm{l}$ of the complexes were added to each well. After 4-5 h of incubation, DMEM medium was replaced by DMEM with $10 \%$ FBS, and all the cells were incubated at $37^{\circ} \mathrm{C}$ in a $\mathrm{CO}_{2}$ incubator for $48 \mathrm{~h}$ prior to further testing.

Western blot analysis. Protein samples were separated with $12 \%$ SDS-polyacrylamide gel (SDS-PAGE) and transferred onto PVDF membranes (Beyotime, Haimen, China). Immune complexes were formed by incubation of the proteins with primary antibodies (R\&D Systems, Minneapolis, MN, USA) overnight at $4^{\circ} \mathrm{C}$. Blots were washed and incubated for $1 \mathrm{~h}$ with HRP-conjugated anti-mouse secondary antibodies (R\&D Systems). Immunoreactive protein bands were detected with an Odyssey Scanning system.

Cell proliferation assay. Cell proliferation was assessed using an MTT assay kit (Sigma, Santa Clara, CA, USA). A total of 4-5 h after miR-497 mimics transfection, cells with various concentrations of miR-497 mimics were trypsinized and counted, respectively. Cells $(1,000)$ were plated in each well of 96-well plates (BD Biosciences, Corning, NY, USA) in triplicate and incubated at $37^{\circ} \mathrm{C}$, and cell proliferation was assessed at 24, 36 and $48 \mathrm{~h}$, following the instructions of the MTT proliferation assay kit. The growth inhibition rate was calculated using the following equation: growth inhibition rate $=[$ the mean optical density (OD) of controls - the mean OD of samples]/the mean OD of controls.

Apoptosis assay. Thirty-six hours after miR-497 mimics transfection, adherent cells were trypsinized. Annexin V incubation reagent $(100 \mu \mathrm{l})$ was prepared by combining: $10 \mu 1$ 10X binding buffer, $10 \mu 1$ propidium iodide (PI), $1 \mu 1$ Annexin V-FITC and $79 \mu \mathrm{l}$ deionized, distilled $\mathrm{H}_{2} \mathrm{O}$. Cells were gently resuspended in the prepared Annexin $\mathrm{V}$ incubation reagent at a concentration of $10^{5}$ to $10^{6}$ cells per $100 \mu \mathrm{l}$. Samples were incubated in the dark for $15 \mathrm{~min}$ at room temperature. All the samples were processed by flow cytometry (FACSCanto ${ }^{\mathrm{TM}}$ II, BD Biosciences, USA). FACS analyses were performed at least 3 times with reproducible results.

Cell cycle assay. Cells were harvested and centrifuged at $1,200 \mathrm{rpm}$ for $5 \mathrm{~min}$ and washed twice in PBS. Subsequently, $3.0 \mathrm{ml}$ ice-cold $70 \%$ ethanol was added dropwise and cells were fixed in this final $70 \%$ ethanol solution for at least $30 \mathrm{~min}$. A total of $250 \mu 10.05 \mathrm{~g} / 1$ PI staining solution was added into each sample and incubated for $30 \mathrm{~min}$ at room temperature and then analyzed by a flow cytometer (FACSCanto ${ }^{\mathrm{TM}}$ II, BD Biosciences). The proliferation index was calculated according to the following equation: $(\mathrm{S}+\mathrm{G} 2 / \mathrm{M}) /(\mathrm{G} 0 / \mathrm{G} 1+\mathrm{S}+\mathrm{G} 2 / \mathrm{M})$. The mock was the control groups treated with lipofect, the negative control (NC) was the control groups transfected with a short RNA sequence. The controls were not treated with miR-497 mimics.

Statistical analysis. Data were expressed as the means \pm standard deviation from at least 3 separate experiments performed 


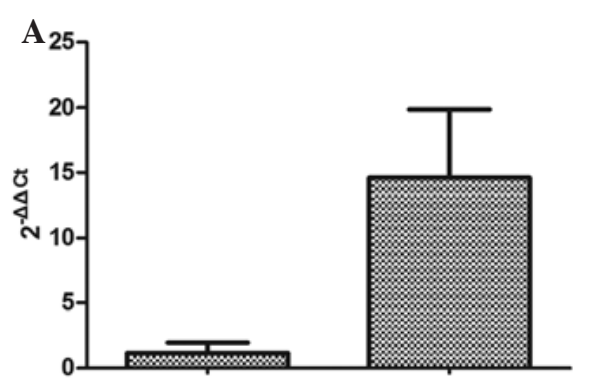

Cancer specimens Normal specimens
B

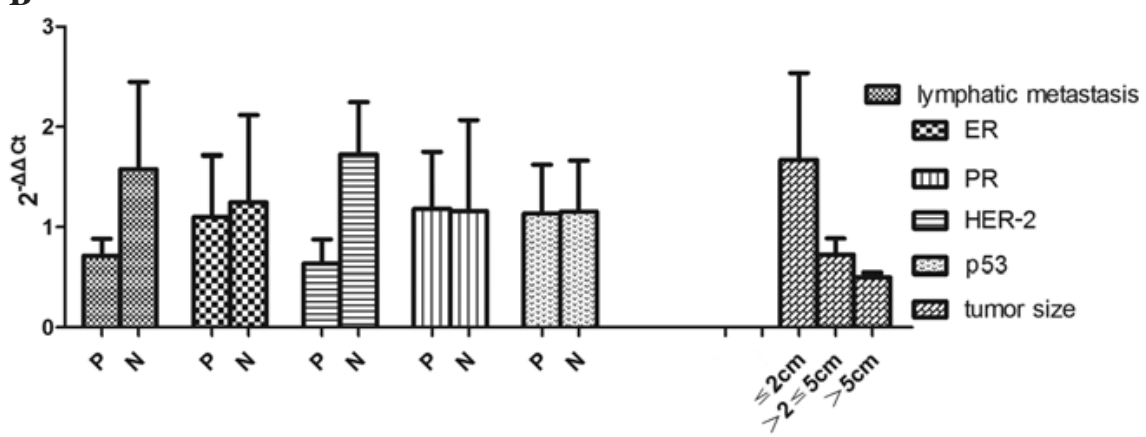

Figure 1. (A) RT-PCR detected a down-regulated expression of miR-497 in breast cancer $(\mathrm{P}<0.01)$. (B) Correlation analysis between miR-497 expression and pathological factors revealed that miR-497 expression was negatively correlated with pathological stage, lymphatic metastasis, tumor size and HER-2. $\mathrm{P}<0.01$. $\mathrm{P}$, positive; N, negative; ER, estrogen receptor; PR, progesterone receptor, HER-2, human epidermal growth factor receptor-2.

in triplicate. Correlations with tumor characteristics were made with Spearman analysis. For RT-PCR analyses, based on the $2^{-\Delta \Delta \mathrm{Ct}}$ method described by Livak and Schmittgen (23), semi-quantitative analysis was used for gene quantification. As variances were not equal, the statistical differences were analyzed using a two independent sample non-parametric test (Mann-Whitney U test) and a K-independent non-parametric sample test (Kruskal-Wallis test) in MCF-7 breast cancer cells, and the null hypothesis was rejected at the 0.05 level.

\section{Results}

Underexpression of miR-497 in breast cancer. The RT-PCR data showed that miR-497 expression was relatively reduced in the breast cancer specimens in comparison to the normal tissues, the relative expression of which was $1.181 \pm 0.779$ and 14.599 $\pm 5.266(\mathrm{P}<0.01)$, respectively (Fig. 1A). Markedly, the abnormal expression pattern of miR-497 was negatively correlated with pathological stage, lymphatic metastasis, tumor size and human epidermal growth factor receptor-2 (HER-2) $(\mathrm{P}<0.01)$. No correlation was observed for miR-497 with estrogen receptor (ER), progesterone receptor (PR) and p53 ( $\mathrm{P}>0.05$ ). No significant expression alteration of miR-198, miR-373 and miR-1289 was found between breast cancer and normal tissues (Fig. 1B).

Suppression of tumor proliferation by miR-497. To explore the potential impact of miR-497 on the proliferation of breast cancer cells, miR-497 mimics were used to interfere with tumor cells at the concentrations of 25, 50, 75, 100 and $125 \mathrm{nmol} / \mathrm{l}$. Cellular viability and proliferation were measured following the protocol of the MTT assay kit at 24, 36 and $48 \mathrm{~h}$. Compared to negative controls, miR-497 significantly repressed the growth of breast cancer cells. Suppression of cell growth by miR-497 was time- and dosage-dependent, and miR-497 at the concentration of $100 \mathrm{nmol} / \mathrm{l}$ and at $36 \mathrm{~h}$ showed the greatest inhibitory effect. As the concentration exceeded $100 \mathrm{nmol} / \mathrm{l}$, no significant alteration of the inhibition rate was observed (Fig. 2).

miR-497 induces the apoptosis of breast cancer cells. To examine whether miR-497 facilitates the apoptosis of breast cancer cells, MCF-7 cancer cells were transfected with

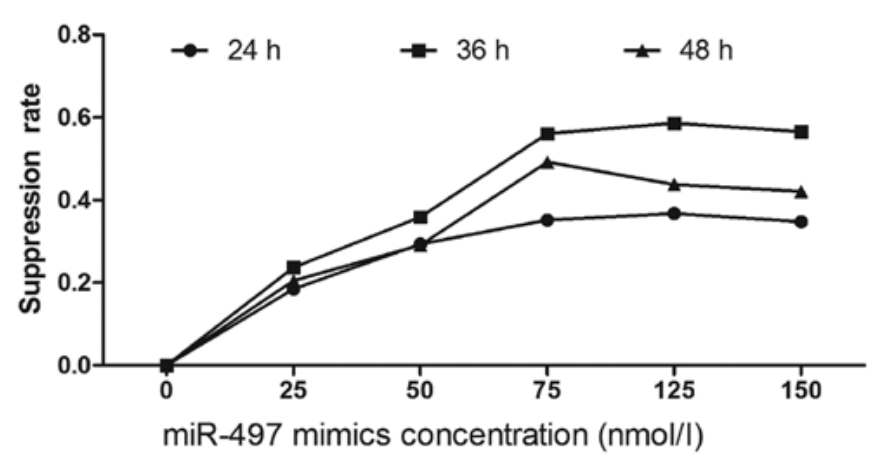

Figure 2. The proliferation suppression rate of negative controls was 0 , and with an increase in the concentration of miR-497 mimics, the suppression rate rose. miR-497 mimics at the concentration of $100 \mathrm{nmol} / \mathrm{l}$ at $36 \mathrm{~h}$ showed the greatest inhibitory effect. All these findings demonstrate that miR-497 inhibits the proliferation of MCF-7 breast cancer cells.

$100 \mathrm{nmol} / \mathrm{l}$ of miR-497 mimics for $36 \mathrm{~h}$. Flow cytometry data indicated that the elevated expression of miR-497 induced early apoptosis, compared to the lipofect-treated controls and the negative controls, and the percentage of early apoptotic cancer cells of the miR-497 treatment groups was markedly increased, which shows that miR-497 can act as an apoptosis inducer in breast cancer in vitro, $\mathrm{P}<0.01, \mathrm{n}=3$ (Fig. 3).

miR-497 disrupts the cell cycle of breast cancer cells. As thoroughly described previously, miR-497 is capable of repressing the proliferation and promote the apoptosis of breast cancer cells. After the transfection of miR-497 mimics at the concentration of $100 \mathrm{nmol} / \mathrm{l}$ for $36 \mathrm{~h}$, flow cytometry analysis revealed that the percentage of $\mathrm{G} 0 / \mathrm{G} 1$ phase cells $(76.23 \pm 1.78 \%)$ dramatically increased in the treatment groups, which was statistically higher than those in the lipofect-treated groups and negative controls $(70.21 \pm 1.52 \%$, $69.81 \pm 1.36 \%$ ), and while the proportion of $\mathrm{S}$-phase cells decreased $(12.79 \pm 0.91 \%)$, the percentage of $\mathrm{G} 2 / \mathrm{M}$ phase cells was not significantly altered. Furthermore, the cellular proliferation index of the treatment groups dropped to 23.76 \pm 0.62 , suggesting that miR-497 can initiate G0/G1 phase arrest and that up-regulation of miR-497 expression leads to the reduction of the S-phase cells as well as the proliferation index (Fig. 4). 


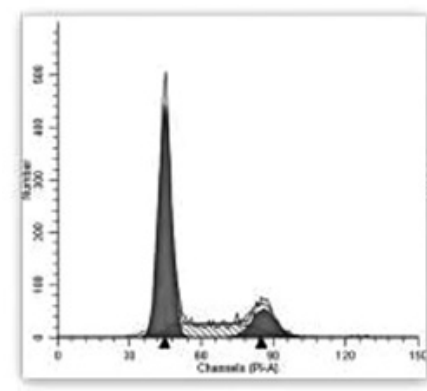

miR-497 mimics

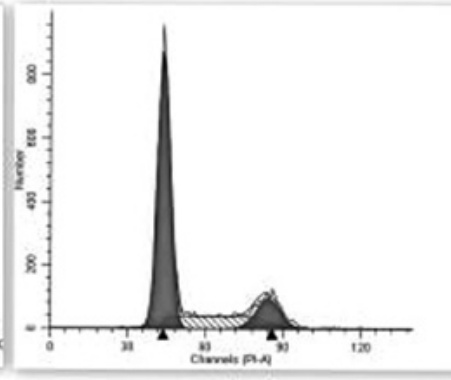

Mock

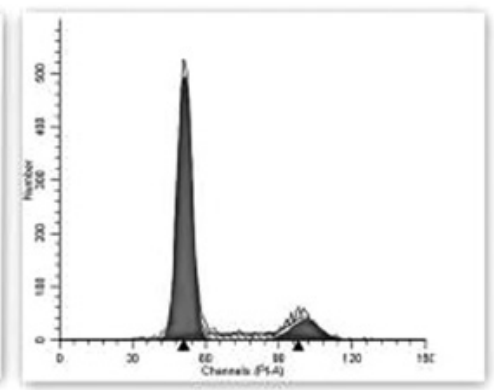

NC

Figure 3. Flow cytometry detected that the percentage of early apoptotic breast cancer cells in thr treatment groups was $10.33 \pm 0.06 \%$, which was statistically different from $2.43 \pm 0.76 \%$ of the lipofect-treated controls and $2.37 \pm 0.12 \%$ of the negative controls $(\mathrm{P}<0.01)$, indicating that miR- 497 induced the apoptosis of breast cancer cells.

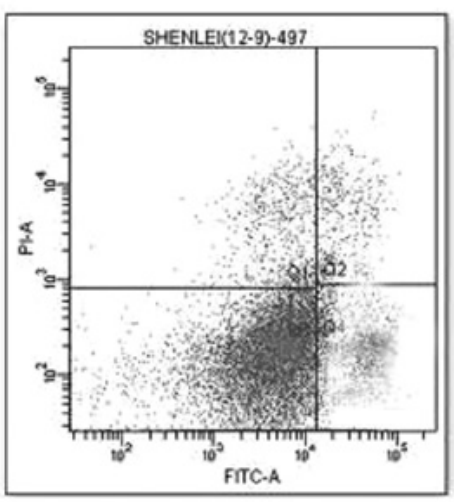

miR-497 mimics

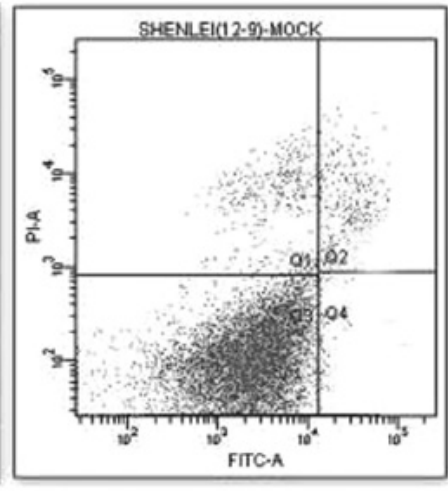

Mock

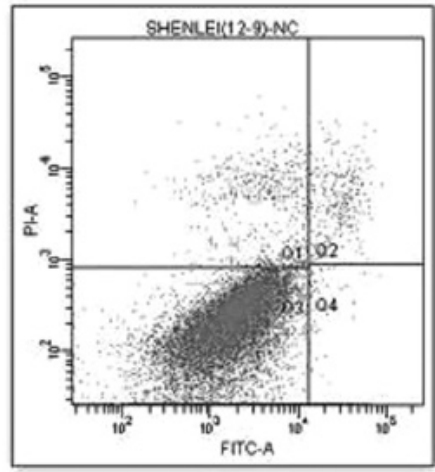

NC

Figure 4. The miR-497 mimic-transfected groups showed an increased percentage of G0/G1 phase cells and a reduction of S-phase cells, suggesting that miR-497 may be able to modulate the cell cycle of MCF-7 breast cancer cells.

A

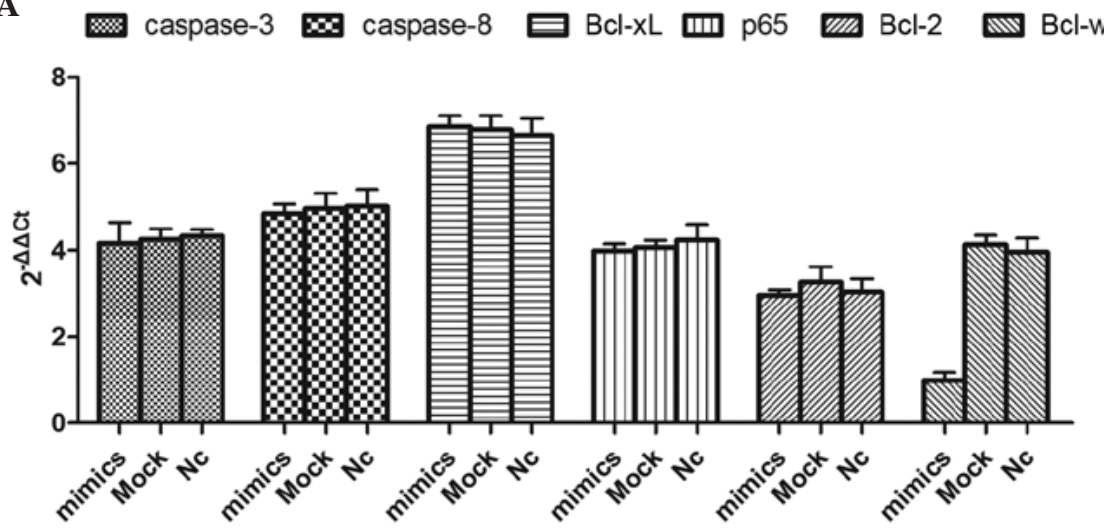

$\mathbf{B}$

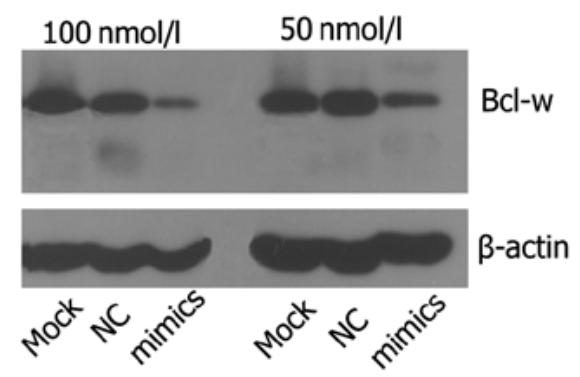

Figure 5. (A and B) Target confirmation by RT-PCR and Western blot analysis showed that miR-497 inhibited the expression of Bcl-w at the mRNA and protein levels, indicating that Bcl-w is the potential downstream target of miR-497. RT-PCR, reverse transcription-polymerase chain reaction.

miR-497 directly targets Bcl-w. To understand the molecular mechanisms by which miR-497 inhibits tumor cell growth and induces cell apoptosis, several members of the apoptotic pathway were selected, including Bcl-w, p65, Bcl-2, Bcl-xL, caspase- 3 and caspase- 8 for further determination of the possible downstream targets. Real-time PCR analysis indicated that Bcl-w expression was significantly different between the miR-497 mimics-transfected groups and lipofect-treated and negative controls, the relative expression of which were $1.004 \pm 0.109,4.303 \pm 0.332$ and $3.971 \pm 0.382$, 
respectively, while no significant alteration of $\mathrm{p} 65, \mathrm{Bcl}-2$, Bcl-xL, caspase-3 and caspase- 8 expression was observed (Fig. 5A). To validate the possibility that miR-497 may target Bcl-w in breast cancer cells, we performed Western blot analysis. In concordance with RT-PCR results, Bcl-w protein expression was significantly decreased with the overexpression of miR-497, suggesting that miR-497 suppresses Bcl-w expression at the protein level. These data demonstrate that miR-497 is capable of inhibiting the growth and facilitating the apoptosis of breast cancer cells via targeting of the proapoptotic gene, Bcl-w (Fig. 5B).

\section{Discussion}

The discovery of the first miRNA, lin-4, in Caenorhabditis elegans symbolized a new era of miRNAs. Their biogenesis, function and potential application have become active areas of research, particularly in cancer. In this study, we selected 4 miRNAs and employed RT-PCR to explore their expression patterns in breast cancer. Compared to the normal breast tissues, miR-497 expression was significantly down-regulated in the breast cancer specimens. Markedly, the miR-497 expression pattern was negatively correlated with pathological stage, lymphatic metastasis, tumor size and HER-2, and no correlation was found between miR-497 and ER, PR and p53. HER-2 is relatively underexpressed in normal breast tissue and overexpressed in $20-30 \%$ of breast cancer tissues. It has been shown that HER-2-positive breast cancer is highly metastatic, proliferative and more likely to relapse. In addition, lymphatic metastasis, higher pathological stage and positive HER-2 are connected to worse prognosis, suggesting that breast cancer patients with elevated expression of miR-497 have better prognosis, and miR-497 may turn out to be a new prognostic marker for breast cancer.

Functional assays have revealed that miR-497 greatly inhibits the cellular growth, induces the apoptosis and disrupts the cell cycle of breast cancer cells. Recently, there has been a large body of evidence for the involvement of miRNAs in the apoptosis of breast cancer. miR-9-3, downregulated in breast cancer, plays a role in the p53-related apoptotic pathway (24). miR-26b impairs the viability and triggers the apoptosis of MCF7 human breast cancer cells by targeting SLC7A11 (25). miR-145 exhibited a pro-apoptotic effect through a death-promoting loop between miR-145 and TP53 (26). The suppression of miR-21 by antisense oligonucleotides hindered tumor cell growth both in vitro and in the xenograft mouse model via the down-regulation of Bcl-2, which consequently increased apoptosis and decreased cell proliferation (14).

Bcl-w, an anti-apoptotic member of the Bcl-2 family, has been demonstrated to be closely associated to cancer formation and progression (27). To date, even though the mutation of Bcl-w has not been verified to be causative of cancer formation, there is evidence that the elevated expression of Bcl-w combined with other oncogenes contributes to tumor occurrence. Target prediction obtained from the target scan showed that the 3'-UTR of Bcl-w contained potential binding sequences complementary to miR- 497 . There are 3 complementary binding sites in the conserved region, and 1 in the non-conserved region, providing a theoretical basis for the target prediction of miR-497. Target validation by RT-PCR and Western blot analysis further confirmed the prediction. Similar to this study, Lin et al described that the up-regulation of miR-122 expression in hepatic cancer led to the decrease of Bcl-w mRNA and protein expression (28).

In conclusion, given the underexpression pattern of miR-497 as well as its potential pro-apoptotic function, it may be concluded that miR-497 acts as a tumor suppressor by targeting Bcl-w in breast cancer. Therefore, the up-regulation of miR-497 artificially offers us a promising new direction for breast cancer treatment in the future.

\section{Acknowledgements}

We give special thanks to all the teachers at the Central Laboratory of Shanghai Tenth People's Hospital for their help and support.

\section{References}

1. WHO fact sheet No.297. World Health Organization. Geneva, 2006.

2. Shi M and Guo N: MicroRNA expression and its implications for the diagnosis and therapeutic strategies of breast cancer .Cancer Treat Rev 35: 328-334, 2009.

3. Le Quesne J and Caldas C: Micro-RNAs and breast cancer. Mol Oncol 4: 230-241, 2010.

4. Iorio MV, Casalini P, Tagliabue E, et al: MicroRNA profiling as a tool to understand prognosis, therapy response and resistance in breast cancer. Eur J Cancer 44: 2753-2759, 2008.

5. Griffiths-Jones S: Annotating noncoding RNA genes. Annu Rev Genomics Hum Genet 8: 279-298, 2007.

6. Bartel DP: MicroRNAs, genomics biogenesis mechanism and function. Cell 116: 281-297, 2004.

7. Breving $\mathrm{K}$ and Esquela-Kerscher A: The complexities of microRNA regulation: mirandering around the rules. Int $\mathrm{J}$ Biochem Cell Biol 42: 1316-1329, 2010.

8. Gregory R I and Shiekhattar R: MicroRNA biogenesis and cancer. Cancer Res 65: 3509-3512, 2005.

9. Volinia S, Calin GA, Liu CG, et al: A microRNA expression signature of human solid tumors defines cacer gene targets.Proc Natl Acad Sci USA 103: 2257-2261, 2006.

10. Iorio M V, Ferracin M, Liu CG, et al: MicroRNA gene expression deregulation in human breast cancer. Cancer Res 65: 7065-7070, 2005.

11. Yan LX, Huang XF, Shao Q, et al: MicroRNA miR-21 overexpression in human breast cancer is associated with advanced clinical stage, lymph node metastasis and patient poor prognosis. RNA 14: 2348-2360, 2008.

12. Yu ZR, Wang CG, Wang M, et al: A cyclin D1/microRNA 17/20 regulatory feedback loop in control of breast cancer cell proliferation. J Cell Biol 182: 509-517, 2008.

13. Wang S, Bian C, Yang Z, et al: miR-145 inhibits breast cancer cell growth through RTKN. Int J Oncol 34: 1461-1466, 2009.

14. Si ML, Zhu S, Wu H, et al: miR-21-mediated tumor growth. Oncogene 26: 2799-2803, 2007.

15. Kong W, He LL, Coppola M, et al: MicroRNA-155 regulates cell survival, growth, and chemosensitivity by targeting FOXO3a in breast cancer. J Biol Chem 285: 17869-17879, 2010.

16. Sachdeva M and Mo YY: MicroRNA-145 suppresses cell invasion and metastasis by directly targeting mucin 1. Cancer Res 70: 378-387, 2010.

17. Valastyan S, Reinhardt F, Benaich N, et al: A pleiotropically acting microRNA, miR-31, inhibits breast cancer metastasis. Cell 137: 1032-1046, 2009.

18. Wu HL, Zhu SM and Mo YY: Suppression of cell growth and invasion by miR-205 in breast cancer. Cell Res 19: 439-448, 2009.

19. Ma L, Teruya-Feldstein J and Weinberg RA: Tumour invasion and metastasis initiated by microRNA-10b in breast cancer. Nature 449: 682-688, 2007.

20. Pan YZ, Morris ME and Yu AM: MicroRNA-328 negatively regulates the expression of breast cancer resistance protein (BCRP/ABCG2) in human cancer cells. Mol Pharmacol 75: 1374-1379, 2009. 
21. Liang ZX, Wu H, Xia J, et al: Involvement of miR-326 in chemotherapy resistance of breast cancer through modulating expression of multidrug resistance-associated protein 1 . Biochem. Pharmacol 79: 817-824, 2010.

22. Chen C, Ridzon DA, Broomer AJ, et al: Real-time quantification of microRNAs by stem-loop RT-PCR. Nucleic Acids Res 33: e179, 2005.

23. Livak KJ and Schmittgen TD: Analysis of relative gene expression data using real-time quantitative PCR and the $2^{-\Delta \Delta \mathrm{Ct}}$ method Methods 25: 402-408, 2001.

24. Hsu PY, Deatherage DE, Rodriguez BA, et al: Xenoestrogeninduced epigenetic repression of microRNA-9-3 in breast epithelial cells. Cancer Res 69: 5936-5945, 2009.

25. Liu XX, Li XJ, Zhang B, et al: MicroRNA-26b is underexpressed in human breast cancer and induces cell apoptosis by targeting SLC7A11. FEBS Lett 585: 1363-1367, 2011.
26. Spizzo R, Nicoloso MS, Lupini L, et al: miR-145 participates with TP53 in a death-promoting regulatory loop and targets estrogen receptor-alpha in human breast cancer cells. Cell Death Differ 17: 246-254, 2010.

27. Bae IH, Park MJ, Yoon SH, et al: Bcl-w promotes gastric cancer cell invasion by inducing matrix metalloproteinase- 2 expression via phosphoinositide 3-kinase, Akt, and Sp1. Cancer Res 66: 4991-4995, 2006

28. Lin CJ, Gong HY, Tseng HC, et al: Mir-122 targets an antiapoptotic gene, Bcl-w, in human hepatocellular carcinoma cell lines. Biochem Biophys Res Commun 375: 315-320, 2008. 